\title{
Análises da evapotranspiração de referência e do índice de aridez para o Nordeste do Brasil
}

\author{
Analysis of reference evapotranspiration and aridity index for Northeast Brazil
}

CABRAL JÚNIOR ${ }^{1}$, J. B.; BEZERRA ${ }^{2}$, B. G.

jorio.cabral@gmail.com;

\begin{abstract}
Resumo
O Nordeste do Brasil (NEB) possui elevados valores de Evapotranspiração de Referência $\left(\mathrm{ET}_{0}\right)$ e déficits de chuvas, que culminam num aspecto de semiaridez predominante. $\mathrm{O}$ objetivo neste trabalho foi analisar a $\mathrm{ET}_{0}$ com as respectivas repercussões para o Índice de Aridez (IA) no NEB. De posse dos dados mensais (1980 a 2013) da $\mathrm{ET}_{0}$ e precipitação, calculou-se o IA preconizado por Thornthwaite (1948). As sub-regiões da $\mathrm{ET}_{0}$ foi determinada por meio da Análise de Cluster. Em seguida, a periodicidade do IA foi analisada para identificar frequências e relações com a $\mathrm{ET}_{0}$, através da análise de Wavelet, com 5\% de significância estatística. Os resultados demonstraram que o NEB apresenta 5 sub-regiões para a $\mathrm{ET}_{0}$, sendo a Sub-região 3 (S3) a que apresentou os maiores valores médios mensal (174,8 $\mathrm{mm}$, em média) e anualmente (2098,0 mm), seguida da S2, S4, S1 e S5 com respectivos valores médios anuais de $1736,9 \mathrm{~mm}, 1643,5$ $\mathrm{mm}, 1489,3 \mathrm{~mm}$, e $1362,8 \mathrm{~mm}$. A primavera obtém predominantemente as maiores $\mathrm{ET}_{0}$ e IA, sobre o NEB. As periodicidades permitiram determinar que a variável dominante para o IA é a precipitação, e que no período seco (periodicidade entre 4 a 8 meses), a ET $_{0}$ não apresenta correlações altas com o IA.
\end{abstract}

Palavras-chave: Agroclimatologia, análise de cluster; análise de Wavelet.

\begin{abstract}
Northeastern Brazil (NEB) has high values of Reference Evapotranspiration $\left(\mathrm{ET}_{0}\right)$ and rainfall deficits, which culminate in a predominant semiarid aspect. The objective of this work was to analyze $\mathrm{ET}_{0}$ with the respective repercussions for the Aridity Index (AI) in NEB. Having the monthly data (1980 to 2013) of $\mathrm{ET}_{0}$ and also precipitation to AI of calculating recommended by Thornthwaite (1948), was calculated on the weather and determined if $\mathrm{ET}_{0}$ the subregions by Cluster Analysis. AI was analyzed to identify relationships with ET0. Periodicities were then determined through the Wavelet analysis, with $5 \%$ of statistical significance. The results showed that the NEB has 5 subregions for the $\mathrm{ET}_{0}$ and subregion 3 (S3), obtains the highest average monthly values (174.8 $\mathrm{mm}$ on average) and annual $(2098.0 \mathrm{~mm})$, then S2, S4, S1 and S5 with respective annual mean values of $1736.9 \mathrm{~mm}, 1643.5 \mathrm{~mm}, 1489.3 \mathrm{~mm}$, and $1362.8 \mathrm{~mm}$. In the spring, the $\mathrm{AI}$ is predominantly greater, on the NEB, and $\mathrm{ET}_{0}$ overcomes the precipitation. The frequencies allowed to determine the dominant variable for the $\mathrm{AI}$ is the precipitation and that the dry period (frequency between 4 to 8 months), the $\mathrm{ET}_{0}$ does not have high correlations with the AI.
\end{abstract}

Keywords: Agroclimatology, cluster analysis; Wavelet analysis.

\section{INTRODUÇÃO}

A evapotranspiração de referência $\left(\mathrm{ET}_{0}\right)$ e o Índice de Aridez (IA) são importantes variáveis climáticas e fundamentais para a compreensão no processo do ciclo hidrológico de uma região. O primeiro representa o potencial da perda de água da superfície para a atmosfera (ALLEN et al., 1998), enquanto que o segundo demonstra a relação existente entre oferta (precipitação) e demanda atmosférica por água (ETo) (THORNTHWAITE, 1948). Ambos são indispensáveis na elaboração e execução de políticas públicas que visam a gestão para o uso eficiente dos recursos hídricos, nas suas variadas aplicações (DROGUE et al., 2004; CHEN et 
al., 2007; HAN; HU, 2012; HAN et al., 2014; SHAN et al., 2015). Além disso, suas complexas interações (por exemplo, déficit de precipitação e excedente de $\mathrm{ET}_{0}$ ) são importantes indicadores de seca (TRENBERTH et al., 2014; WANG et al., 2016).

Para Liu et al. (2013) a seca se inicia pela falta de chuva durante um longo período de tempo. Entretanto esse fenômeno é intensificado se houver uma elevada perda de água para a atmosfera $\left(\mathrm{ET}_{0}\right)$. Diversos estudos têm relatado essa vulnerabilidade em relação às irregularidades climáticas, com ênfase aos déficits hidroclimáticos (MARES et al., 1985; AMBRIZZI et al., 2007; IPCC, 2007; PAREDES et al., 2015; MARENGO et al., 2017). No semiárido do NEB, por exemplo, a escassez hídrica é crônica e contínua, mesmo assim a população ainda não se adaptou ao fenômeno (MARENGO et al., 2011).

A seca afeta mais pessoas do que qualquer outro desastre natural (KEYANTASH; DRACUP, 2002; MARENGO et al., 2017). Somado a isso, no semiárido do NEB, a população rural mais pobre é a mais vulnerável a este fenômeno climático (MARENGO et al., 2017). O agravante da seca na região afeta principalmente a vazão dos rios, impactando o abastecimento de água e a produção de energia (MARENGO et al., 2011).

Para o NEB, estudos realizados por Cunha et al. (2015) verificaram que a seca de 20122013 afetou 85\% da cobertura vegetal na região semiárida. Brito et al. (2017) identificaram que ocorreu uma seca grave e prolongada entre 2011-2015, no período analisado de 1981 a 2015, e afirmaram ser a seca mais frequente e grave com 6295 declarações de calamidade decretadas pelo governo federal; foram 33,4 milhões de pessoas atingidas, durante 2012 a 2016, e um dano de $\mathrm{R} \$ 104$ bilhões em investimentos públicos para mitigar impactos decorrentes da seca (MI, 2017). Marengo e Bernasconi (2015), analisaram índices de aridez/seca, através de modelos climáticos, e projetaram uma expansão geográfica com aumento desses índices.

Esses resultados citados foram avaliados, respectivamente, por meio de sensoriamento remoto para a vegetação. Por índices de precipitação e de vegetação. Também por registros de danos por órgãos públicos. E ainda por estimativas de IA através de modelos climáticos. Vale destacar que um dos IA e/ou de seca, mais usual é o Índice de Precipitação Padronizado (SPI) (MCKEE et al., 1993), porém esse método é baseado somente na precipitação e, embora seja muito útil como medida de déficit de precipitação ou seca meteorológica, é limitado porque não considera a $\mathrm{ET}_{0}$ (TRENBERTH et al., 2014).

Uma melhor compreensão das características espaciais e temporais da $\mathrm{ET}_{0}$ e IA, além de ser importante indicador climático, é necessária para o uso eficiente dos recursos hídricos em seus múltiplos usos (agricultura, abastecimento humano, produção de energia, etc). Diante 
do exposto o objetivo principal deste trabalho é analisar características da $\mathrm{ET}_{0}$ e do IA, para o $\mathrm{NEB}$, e identificar por sub-regiões homogêneas da $\mathrm{ET}_{0}$ mensal, suas respectivas relações.

\section{MATERIAL E MÉTODOS}

\section{1. Área de estudo}

A área de estudo está localizada no Nordeste Brasileiro (NEB), essa região é a segunda mais populosa do Brasil, com 57,36 milhões de habitantes, e a terceira maior em dimensão territorial, com 1.554,291 $\mathrm{Km}^{2}$ (IBGE, 2017). Encontra-se inserida nos trópicos $\left(1^{\circ} \mathrm{a} 18^{\circ} \mathrm{S} ; 35\right.$ a $47^{\circ} \mathrm{W}$ ). Apresenta $60 \%$ dos registros de desastres naturais do Brasil, em função da seca (CEPED/UFSC, 2013), sendo que desde 1980 contabilizaram-se mais de 50 milhões de pessoas afetadas por esse fenômeno (SAPIR; BELOW, 2014). Atualmente 92,8\% dos municípios que compõem a região semiárida do Brasil está inserida no NEB.

Os principais biomas que compõe o NEB são: Caatinga, Mata Atlântica, Amazônia e Cerrado (IBGE, 2004). A precipitação acumulada anual, em média, varia de $300 \mathrm{~mm}$ (no Bioma Caatinga) a mais de 1.500 mm (nos Biomas Amazônicos e da Mata Atlântica) (NIMER, 1989), e os principais sistemas meteorológicos causadores de chuvas na região são: a Zona de Convergência Intertropical (ZCIT), Vórtice Ciclônico de Altos Níveis (VCAN), Distúrbios Ondulatórios de Leste (DOL), Complexo Convectivo de Mesoescala (CCM), Zona de Convergência do Atlântico Sul (ZCAS), Linhas de Instabilidades (LI) (FERREIRA; MELLO, 2005; REBOITA et al., 2010; MARENGO et al., 2011; HASTENRATH, 2012). Na região semiárida do NEB, o período chuvoso ocorre entre março e maio, enquanto parte leste o máximo de chuva ocorre entre junho e julho (HASTENRATH, 1990; XAVIER, 2001; NOBRE et al., 2006; ALMEIDA; CABRAL JÚNIOR, 2014).

A localização da área de estudo e a distribuição do número de pontos de grade (2043) podem ser observados na Figura 1. Os pontos de grade referem-se aos pontos com coordenadas geográficas fixas e são equidistantes $\left(0,25^{\circ} \times 0,25^{\circ}\right)$, em cada ponto há uma série temporal de dados de $\mathrm{ET}_{0}$ e precipitação. Esses dados foram analisados em todo o NEB, cuja região encontra-se no Nordeste da américa do Sul. Destaca-se que nove estados compõem essa região, sendo: Maranhão (MA), Piauí (PI), Ceará (CE), Rio Grande do Norte (RN), Paraíba (PB), Pernambuco (PE), Alagoas (AL), Sergipe (SE) e Bahia (BA). 


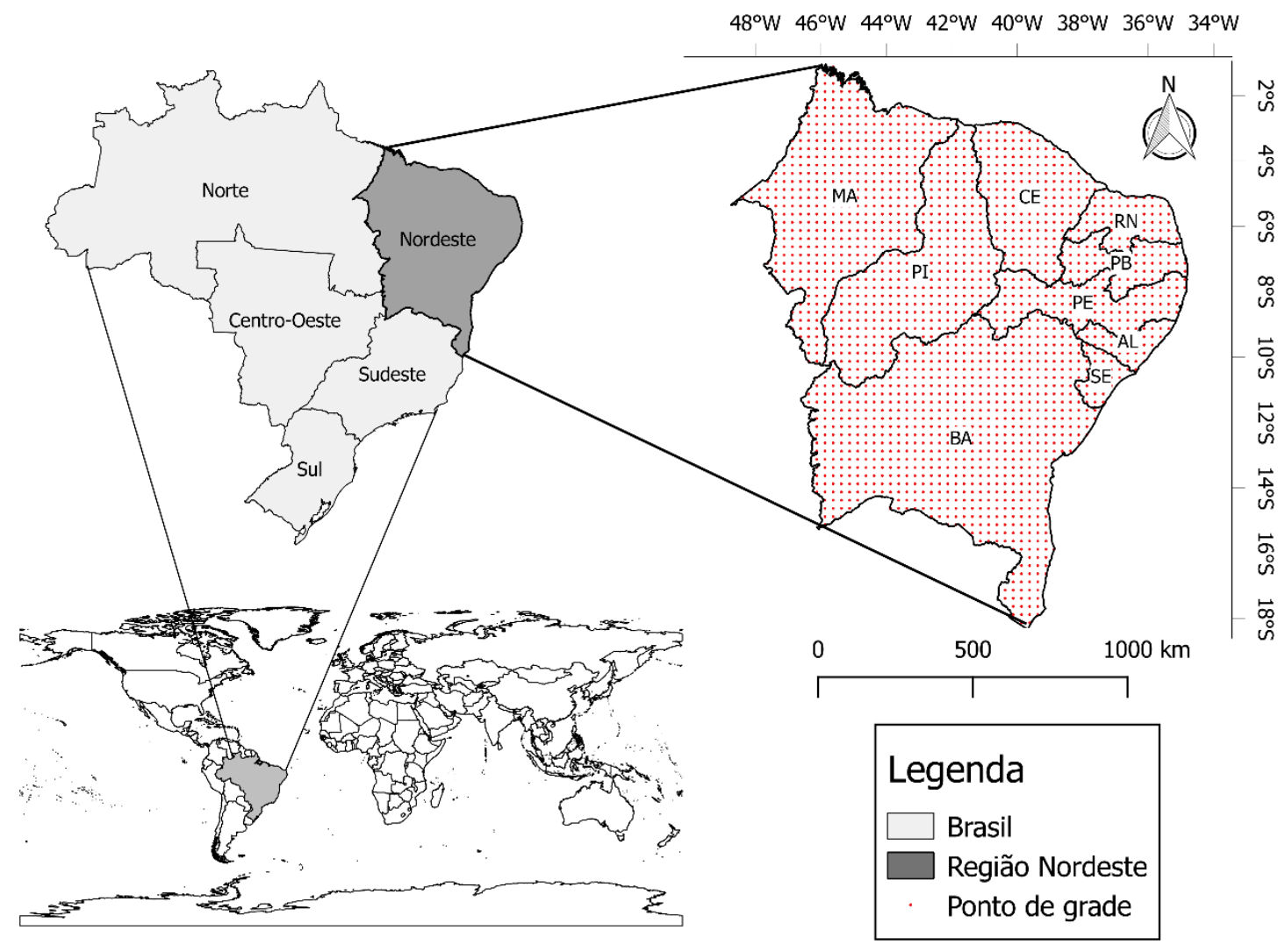

Figura 1. Localização da região Nordeste do Brasil e seus respectivos estados, com os pontos de grades distribuídos espacialmente em $0,25^{\circ}$ x $0,25^{\circ}$ referentes aos dados de precipitação e $\mathrm{ET}_{0}$. Fonte: Elaboração dos autores.

\subsection{Dados}

Os dados utilizados neste trabalho foram disponibilizados por Xavier et al. (2015), por meio de um banco de dados distribuídos espacialmente, cujo espaçamento de grade é $0,25^{\circ} \mathrm{x}$ $0,25^{\circ}$, aproximadamente $28 \mathrm{~km}^{2}$, cada ponto possui uma série temporal mensal de 1980 a 2013.

Esses autores utilizaram uma série de dados meteorológicos de estações oficiais de diferentes instituições brasileiras: Instituto Nacional de Meteorologia (INMET), Agência Nacional de Águas (ANA) e Departamento de Águas e Energia Elétrica de São Paulo (DAEE). Em seguida testaram diferentes métodos de interpolação, para as escalas espacial e temporal, e validaram os respectivos preenchimentos.

Os dados utilizados referentes à $\mathrm{ET}_{0}$, foram estimados pelo método padrão internacional de Penman-Monteith (ALLEN et al., 1998), expressa pela equação 1.

$$
E T_{0}=\frac{0,408 \cdot \Delta\left(R_{n}-G\right)+\gamma\left(\frac{900 \cdot U_{2}}{T_{\text {med }}+273}\right) D P V}{\Delta+\gamma \cdot\left(1+0,34 U_{2}\right)}
$$


em que $\Delta$ é a inclinação da curva de pressão de vapor $\left(\mathrm{KPa}^{\circ} \mathrm{C}^{-1}\right)$; $\mathrm{Rn}$ é o saldo do balanço de radiação solar incidente em ondas curtas e a radiação terrestre, emitida em ondas longas; G é a densidade de fluxo de calor do solo (tanto em $\mathrm{MJ} \mathrm{m}^{-2} \mathrm{~d}^{-1}$ ); $\gamma$ é a constante psicrométrica ( $\mathrm{kPa}$ $\left.{ }^{\circ} \mathrm{C}^{-1}\right) ; T_{\text {med }}$ é a temperatura média diária do $\operatorname{ar}\left({ }^{\circ} \mathrm{C}\right) ; U_{2}$ é a velocidade média do vento medida a $2 \mathrm{~m}$ de altura $\left(\mathrm{m} \cdot \mathrm{s}^{-1}\right) ; D P V$ é o déficit de pressão de vapor $(\mathrm{kPa})$.

A partir dos dados de $\mathrm{ET}_{0}$, utilizou-se também os dados de precipitação para em seguida obter-se o IA utilizando-se a equação proposta por Thornthwaite (1948), que considera oferta (precipitação) e demanda $\left(\mathrm{ET}_{0}\right)$ da água em nível de superfície. Esse autor definiu o IA para expressar o grau árido em áreas áridas ou semiáridas, através da equação 2.

$$
I A=\frac{\left(E T_{0}-P\right)}{E T_{0}}
$$

Quando não houver precipitação, o IA será igual a 1 (grau de aridez mais elevado), contrariamente se $P$ for igual ou superior a $E T_{0}$, o $I A$ será respectivamente igual a zero ou negativo (neste caso, não há aridez aparente). Neste trabalho, o IA foi analisado nos intervalos de tempo sazonal e espacial para o NEB.

\subsection{Procedimentos metodológicos}

As etapas para realização deste trabalho, Figura 2, consistiram inicialmente em delimitar a área de estudo (NEB) e na aquisição dos bancos de dados que estão disponíveis ao público (https://utexas.app.box.com/v/Xavier-etal-IJOC-DATA).

De posse dos dados, foram determinadas sub-regiões homogêneas de acordo com a variação média mensal da $\mathrm{ET}_{0}$ (Clusters) utilizando a técnica de Análise de Agrupamento (MINGOTI, 2005). Após a definição das sub-regiões, analisou-se individualmente cada uma, utilizando-se estatísticas descritivas (média, desvio padrão, coeficiente de variação, valor máximo e valor mínimo).

A periodicidade da $\mathrm{ET}_{0}$ versus IA, mensal e anual, foi analisada utilizando a técnica de Análise Wavelets. A referida análise foi utilizada para identificar o comportamento dessas duas importantes variáveis hidroclimáticas, em séries temporais em domínios de tempofrequências (ciclos) e correlação. 


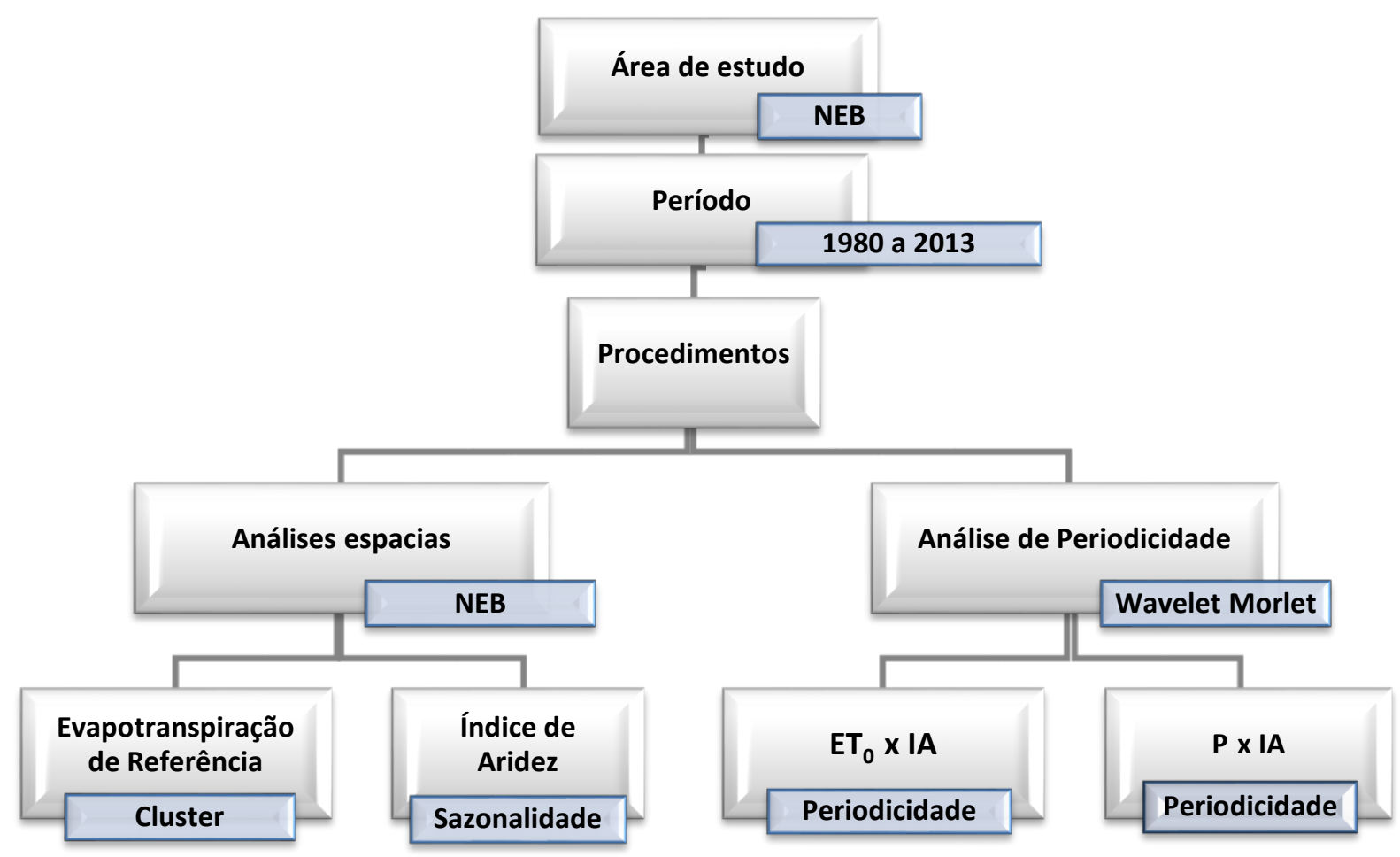

Figura 2. Organograma hierárquico das etapas para realização da pesquisa. Fonte: Elaboração dos autores.

\subsection{Análise de Agrupamento (Cluster)}

A análise de Cluster ou análise de agrupamento é um método estatístico multivariado, que consiste em dividir elementos da amostra ou população em grupos, de forma que os elementos pertencentes a um mesmo grupo sejam similares entre si expressando as variáveis envolvidas, e os elementos em grupos diferentes sejam heterogêneos em relação a estas mesmas variáveis (MINGOTI, 2005). O objetivo é maximizar a similaridade entre os elementos dentro do grupo e de forma concomitante potencializar a heterogeneidade existente entre eles.

A caracterização de cada sub-região, para a $\mathrm{ET}_{0}$, foi realizada utilizando-se estatísticas descritivas e, simultaneamente realizaram-se comparações entre as sub-regiões da $\mathrm{ET}_{0}$ com os valores do IA no intuito de compreender, por exemplo, relações quantitativas entre o IA com as respectivas variáveis envolvidas no seu cálculo (ETo e precipitação).

O processo para o agrupamento consistiu no cálculo de uma medida de dissimilaridade, que de acordo com Mimmack et al. (2001) uma das medidas mais indicadas para a regionalização de dados climáticos, é o método da distância euclidiana, expressa por: 


$$
\mathrm{d}\left(X_{i}, X_{j}\right)=\left[\sum_{k=1}^{p}\left(X_{i, k}-X_{j, k}\right)^{2}\right]^{\frac{1}{2}}
$$

Sendo, $X_{i, k}$ e $X_{j, k}$ os elementos a serem comparados, $X_{i} \neq X_{j}=1, \ldots, n$ (número total da amostra), portanto representam os dois elementos a serem comparados, de acordo com a observação da K-ésima variável de cada elemento amostral e $p$ representa o número de variáveis. De acordo com Mingoti (2005) são realizadas comparações entre os dois elementos amostrais para cada variável pertencente ao vetor de observações.

Neste trabalho utilizou-se a abordagem hierárquica, para construção dos clusters, cujo método de ligação adotado foi o de Ward (variância mínima), proposto por Ward (1963), que se fundamenta nos princípios da análise de variância. Em cada passo do método foi calculada a soma de quadrados dentro de cada grupo. Esta soma é o quadrado da distância euclidiana de cada elemento pertencente ao grupo em relação ao correspondente vetor de médias, de acordo com a equação:

$$
\mathrm{w}=\sum_{g=1}^{G} \sum_{i=1}^{n_{g}}\left\|X_{i}-\bar{X}_{g}\right\|^{2}=\sum_{g=1}^{G} \sum_{i=1}^{n_{g}} \sum_{k=1}^{k}\left(X_{i, k}-\bar{X}_{g, k}\right)^{2}
$$

Sendo, $W$ - representa a função de ligação de Ward, dada pela soma de quadrados dentro de cada grupo $\left(G_{i}\right)$ (medida de homogeneidade); $G$ - o número de elementos no grupo $G_{i}$ quando se está no passo $k$ do processo de agrupamento; $X_{i, k}$ - o vetor de observações do k-ésimo elemento que pertence ao i-ésimo grupo; $\bar{X}_{g}$ - o centróide do grupo $G_{i}$.

Em virtude das propriedades do método hierárquico é possível construir um gráfico chamado dendrograma (TIMM, 2002), ou seja, um diagrama bidimensional que possui forma de árvore cujo eixo vertical representa o nível de similaridade (ou dissimilaridade) e no eixo horizontal, são representados os elementos amostrais. A escolha do número de grupos $(g)$ é feita a partir do dendrograma de maneira subjetiva (KELLER FILHO et al., 2005), no entanto, essa subjetividade requer um conhecimento prévio sobre a estrutura dos dados.

\subsection{Análise de Wavelet de Morlet}

A periodicidade da $\mathrm{ET}_{0}$ e do IA, durante os últimos 34 anos, sobre cada uma das subregiões previamente definidas, foram analisadas utilizando-se a técnica de Wavelet. Para este fim, está técnica tem sido utilizada em estudos climáticos (WANG et al., 2014; ZHANG et al., 
2015; WANG et al., 2017; FENG et al., 2017; XU et al., 2017). A técnica de Wavelet, também conhecida como ondeletas, é eficiente para análises em escala multi-temporal (por exemplo, mensal, sazonal, anual e decadal). A função de Wavelet $(\psi(t))$, o coeficiente da transformada de WaveLet $\left(W_{f}(a, b)\right)$ e a onda contínua $\left(\psi_{a, b}(t)\right)$ foram calculados, respectivamente, de acordo com as equações 5,6 e 7 .

$$
\begin{aligned}
& \psi(t)=e^{i c t} e^{-t^{2} / 2} \\
& W_{f}(a, b)=|a|^{-\frac{1}{2} \int_{t=-\infty}^{\infty} \int(x) \bar{\psi}\left(\frac{t-b}{a}\right) d t}=\left\langle\int(x), \psi_{a, b}(t)\right\rangle \\
& \psi_{a, b}(t)=|a|^{-\frac{1}{2}} \psi\left(\frac{t-b}{a}\right) a, b \in R, a>0
\end{aligned}
$$

Em que, $\psi(t)$ : é a Wavelet principal; $c$ - é uma constante (igual a 6,2); $(a, b)$ - são os coeficientes da transformada de Wavelet; $\langle$,$\rangle - é o produto interno; a$ - é o fator de escala que representa o comprimento do ciclo (periodicidade); $b$ - é um fator de tempo que representa o intervalo do tempo; $\psi_{a, b}(t)$ - é a onda contínua; $\operatorname{Var}(a)$ - é a variância da onda.

A correlação ou coerência dos sinais de frequência de tempo entre as séries em estudo, foi obtida através da Coerência Wavelet. Interpreta-se como medida de coerência duas ondas simples no tempo-frequência, onde, a quantidade de variabilidade de uma determinada variável pode ser explicada pela variabilidade de outra (TORRENCE; WEBSTER, 1999). Os valores de correlações resultantes são plotados nos gráficos, em módulo, variando de 0 a 1 , quanto mais próximo de 1 maior é a correlação entre os sinais.

Os cálculos e análises neste trabalho foram desenvolvidos, considerando-se uma significância estatística de 5\%, análises estas utilizando-se o software estatístico livre R, versão 3.3.1. Para representação gráfica foram construídos mapas utilizando-se o software livre Quantum Gis (QGIS), versão 2.14.11.

\section{RESULTADOS E DISCUSSÃO}

A análise de cluster possibilitou a divisão de sub-regiões com características homogêneas no NEB, considerando-se a média da $\mathrm{ET}_{0}$ mensal. Percebe-se, na Figura 3-A, que após o número de cluster 5 a soma dos quadrados permaneceu praticamente constante, portanto esse foi o número considerado para subdividir o NEB em cinco sub-regiões, cujos pontos de grade foram agrupados e demonstrados através do dendrograma (Figura 3-B), com o respectivo 
recorte (linha vermelha) utilizado para dividir o número de grupos a partir do método da distância euclidiana e do agrupamnto hierárquico através da técnica de ligação de Ward.
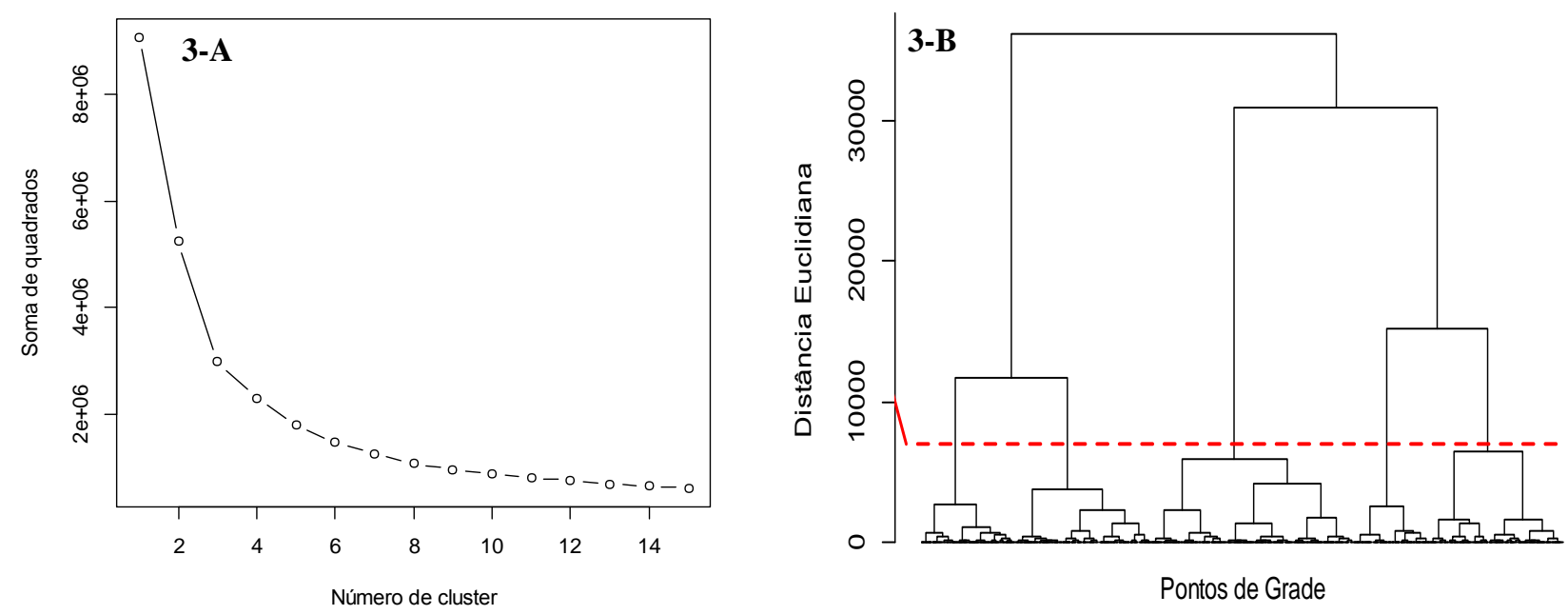

Figura 3. Número de cluster (3-A) e dendrograma (3-B) referentes as cinco sub-regiões da evapotranspiração de referência, média do acumulado mensal, a partir da análise de agrupamento, considerando-se a distância euclidiana e o método de ligação de Ward, para o Nordeste do Brasil, período: 1980 a 2013. Fonte: Elaboração dos autores.

A representação espacial das cinco sub-regiões sobre o NEB, são representadas na Figura 4-A, cujas médias mensais e estatísticas anuais são identificadas, respectivamente, na Figura 4-B e Tabela 1.

Verificou-se que a Sub-região 3 (S3) apresentou os maiores valores de $\mathrm{ET}_{0}$ mensal (Figura 4-B) e consequentemente anual (Tabela 1), com maiores valores registrados na estação da primavera, média de 213,3 mm.mês ${ }^{-1}$, e a partir desse período ocorre uma diminuição gradativa até atingir o valor mínimo no início do solstício de inverno (junho), com média de 129,4 mm.mês ${ }^{-1}$. Destaca-se ainda que a S3 também apresentou a maior variabilidade, com acumulado médio anual de 2098,0 mm e desvio padrão de $164,7 \mathrm{~mm}$, ou seja, 7,9\% de variação em relação a média (Tabela 1).

A S2 é a segunda com maiores valores médios mensais e anuais, o que a difere da S3, além da magnitude, é a característica observada no primeiro semestre, cujas médias e variações mensais são menores $(123,4 \pm 6,5 \mathrm{~mm}, \mathrm{CV}=5,2 \%)$ em relação aos valores observados no mesmo semestre para a S3 $(153,7 \mathrm{~mm} \pm 21,2 \mathrm{~mm}, \mathrm{CV}=21,2 \%)$. Ressalta-se que as S3 e/ou S2 contemplam parcialmente todos os estados do NEB, exceto Alagoas e Sergipe, Figura 4-A. 

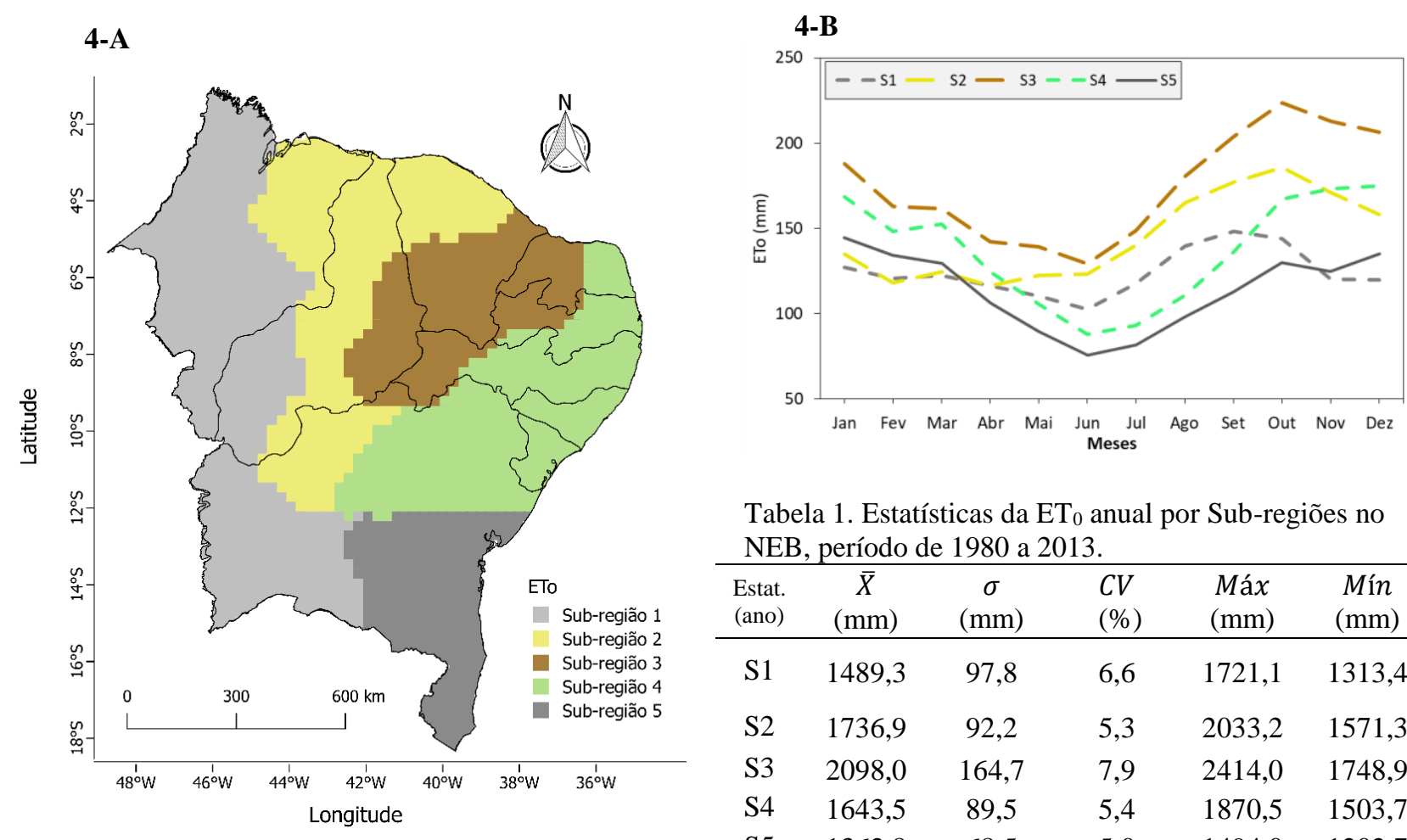

Tabela 1. Estatísticas da $\mathrm{ET}_{0}$ anual por Sub-regiões no NEB, período de 1980 a 2013.

\begin{tabular}{cccccc}
\hline $\begin{array}{c}\text { Estat. } \\
\text { (ano) }\end{array}$ & $\begin{array}{c}\bar{X} \\
(\mathrm{~mm})\end{array}$ & $\begin{array}{c}\sigma \\
(\mathrm{mm})\end{array}$ & $\begin{array}{c}C V \\
(\%)\end{array}$ & $\begin{array}{c}\text { Máx } \\
(\mathrm{mm})\end{array}$ & $\begin{array}{c}\text { Mín } \\
(\mathrm{mm})\end{array}$ \\
\hline S1 & 1489,3 & 97,8 & 6,6 & 1721,1 & 1313,4 \\
S2 & 1736,9 & 92,2 & 5,3 & 2033,2 & 1571,3 \\
S3 & 2098,0 & 164,7 & 7,9 & 2414,0 & 1748,9 \\
S4 & 1643,5 & 89,5 & 5,4 & 1870,5 & 1503,7 \\
S5 & 1362,8 & 68,5 & 5,0 & 1494,0 & 1202,7 \\
\hline
\end{tabular}

Figura 4. Sub-regiões da ET 0 distribuídas na região Nordeste do Brasil (4-A), com as respectivas médias mensais acumuladas (4-B) e estatísticas dos acumulados anuais (Tabela 1), período de 1980 a 2013. Fonte: Elaboração dos autores.

Por outro lado, a S5, contemplada pela região sudeste da Bahia, Figura 4-A, apresentou os menores valores mensais, de abril a outubro (99,0 mm.mês ${ }^{-1}$, em média), e consequentemente anual com 1362,8 $\mathrm{mm}$ (Tabela 1). As variabilidades, na S5, também foram as menores e registraram um desvio padrão de $68,5 \mathrm{~mm}$ e $5 \%$ de coeficiente de variação (Tabela 1). A amplitude dos acumulados anuais também foi a menor, com 292,0 mm.

As S5 e S4 (Figura 4-A) apresentam picos de $\mathrm{ET}_{0}$ mensais observados no verão (Figura 4-B), diferentes das demais sub-regiões que apresentaram valores máximos na estação da primavera. Ambas apresentaram também menores amplitudes mensais, e isso pode estar relacionado a baixa amplitude de umidade relativa, uma vez que nessas duas sub-regiões os ventos predominantes são os alísios de sudeste, esses ventos transportam umidade do atlântico e contribuem para que a costa leste do NEB seja uma entre as áreas mais úmidas da região. Entretanto, ressalta-se que devido ao grande volume de dados, a S5 e S4 podem ter sido potencializadas pela análise de cluster, quando se esperaria uma faixa mais estreita de ambas próximo a costa litorânea. Quando se compara essas duas sub-regiões, o que basicamente as diferenciam é a magnitude nos respectivos valores, tanto em termos mensais (Figura 4-B) quanto anuais (Tabela 1), a $\mathrm{ET}_{0}$ na $\mathrm{S} 4$ é cerca de 20,6\% maior que a $\mathrm{ET}_{0}$ na $\mathrm{S} 5$. 
A S1 apresentou os menores valores médios da $\mathrm{ET}_{0}$ no verão $\left(122,5 \mathrm{~mm} . \mathrm{mês}^{-1}\right)$, seguida pela S2 (137,1 mm.mês $\left.{ }^{-1}\right)$, S5 (mm.mês $\left.{ }^{-1}\right)$, S4 (138,0 mm.mês $\left.{ }^{-1}\right)$ e S3 (164,0 mm.mês $\left.{ }^{-1}\right)$.

Para os elevados valores da $\mathrm{ET}_{0}$, cerca de dois mil milímetros por ano em diferentes localidades do NEB, corroboram com os resultados encontrados por outros autores, entre eles destacam-se os trabalhos realizados por Silva (2004), Santos et al. (2010), Jesus e Mattos (2013) e entre outros, especialmente em localidades dentro da S3 definida neste trabalho. Enquanto que para as variabilidades sazonais da $\mathrm{ET}_{0}$ serem predominantemente maiores na primavera e menores no inverno, estão de acordo com os resultados encontrados por Oliveira et al. (2014), Cabral Júnior (2015), Cabral Júnior et al. (2017).

Os elevados valores de $\mathrm{ET}_{0}$ podem contribuir na intensificação de secas, principalmente em regiões áridas e semiáridas. Por isso inseriu-se neste trabalho a variável precipitação para quantificar as principais características do IA no NEB. Na Figura 5 são observadas as variabilidades espaciais e sazonais: Dezembro-Janeiro-Fevereiro (DJF), Março-Abril-Maio (MAM), Junho-Julho-Agosto (JJA) e Setembro-Outubro-Novembro (SON). 

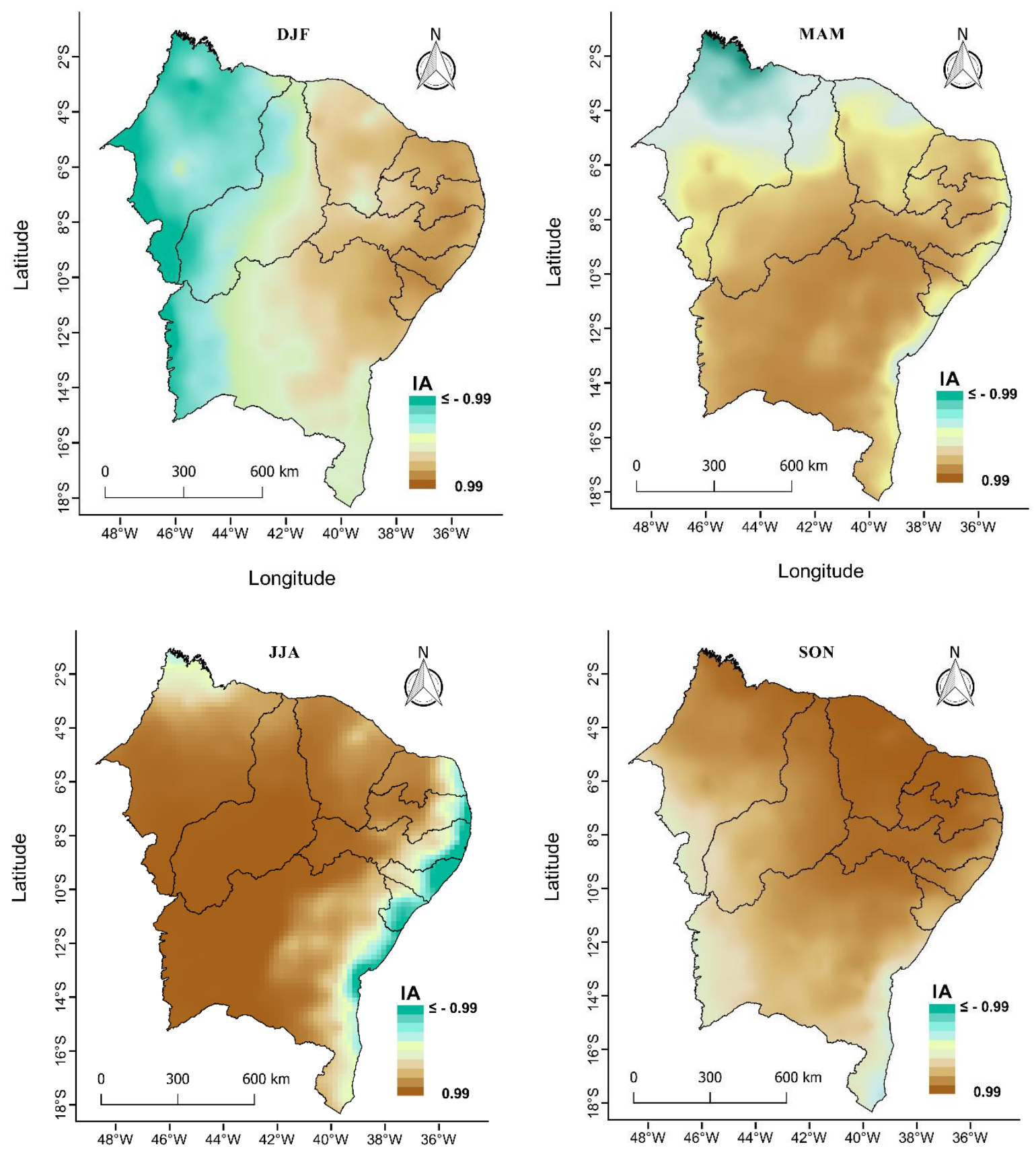

Longitude

Longitude

Figura 5. Variabilidade espacial e sazonal do Índice de Aridez para a região Nordeste do Brasil no período de 1980 a 2013. Fonte: Elaboração dos autores.

Percebe-se que os maiores valores do IA ocorrem em SON (primavera), cujos valores aproximam-se do valor 1,0. Para esse período, além da influência dos maiores valores da $\mathrm{ET}_{0}$, mencionados nas Figuras 4-A e 4-B, coincide de ser o período menos chuvoso, principalmente na região semiárida, quando os valores médios mensais de chuvas aproximam-se de zero (OLIVEIRA et al., 2013a; ALMEIDA; CABRAL JÚNIOR, 2014; REBOITA et al., 2016), isso expressa, portanto, o período mais seco do ano para o NEB (menor precipitação e maior demanda atmosférica). 
A influência da precipitação no IA fica mais visível nas demais estações do ano (DJF, MAM e JJA). Por exemplo, em DJF a parte oeste do NEB é a mais chuvosa cujos IA foram negativos e influenciados pelas precipitaçãoes superiores à $\mathrm{ET}_{0}$. De acordo com Reboita et al. (2010) essas precipitações são ocasionadas principalmente pelos VCAN, ZCIT, CCM e LI. Para a configuração do IA no outono (MAM), marcada pelos valores negativos ao norte do NEB, é determinada pela atuação da ZCIT, que nesse período encontra-se mais incursionada para o Hemisfério Sul e modula a estação chuvosa no norte do NEB (UVO, 1989; MOLION; BERNARDO, 2002; SOUZA; CAVALCANTI, 2009; MARENGO et al., 2011). Nessa estação, o IA médio fica inferior a 0,2, e mesmo sendo o período mais chuvoso, por exemplo, nas microrregiões do seridó (paraibano e potiguar), cariri paraibano e o sudoeste do Ceará, a $\mathrm{ET}_{0}$ supera a precipitação, em média, e consequentemente, o IA registra valores positivos.

Em JJA o extremo noroeste do estado do Maranhão e principalmente a costa leste do NEB apresentam os menores IA, com valores negativos (Figura 5). Na costa leste da S4 (Figura 4-A), esse comportamento deve-se a influência principal dos DOL que de acordo com Oliveira et al. (2013b) e Amorim et al. (2014) esse sistema provoca chuvas no inverno e é o principal causador dos maiores acumulados de precipitação para essa região.

Na Figura 6 (A e B) são verificadas as periodicidades mensais das correlações existentes entre a $\mathrm{ET}_{0}$ com o IA (6A) e a precipitação com o IA (6B) para a S3 definida na Figura 4, essa sub-região foi escolhida para análise devido a sua característica de maiores magnitudes e variabilidades nos valores da $\mathrm{ET}_{0}$. Pode-se verificar, Figura 6-A, que houve uma frequência predomintemente maior de eventos na periodicidade de 8 a 16 meses com correlações positivas (indicativo da seta para a direita) com significância estatística de 5\%. O contrário ocorreu quando se observou a relação existente entre precipitação e IA, Figura 6-B, com correlações negativas (indicativo da seta para a esquerda) e elevadas em todos os intervalos de periodicidade (4 a 128 meses), significativas a $5 \%$. 

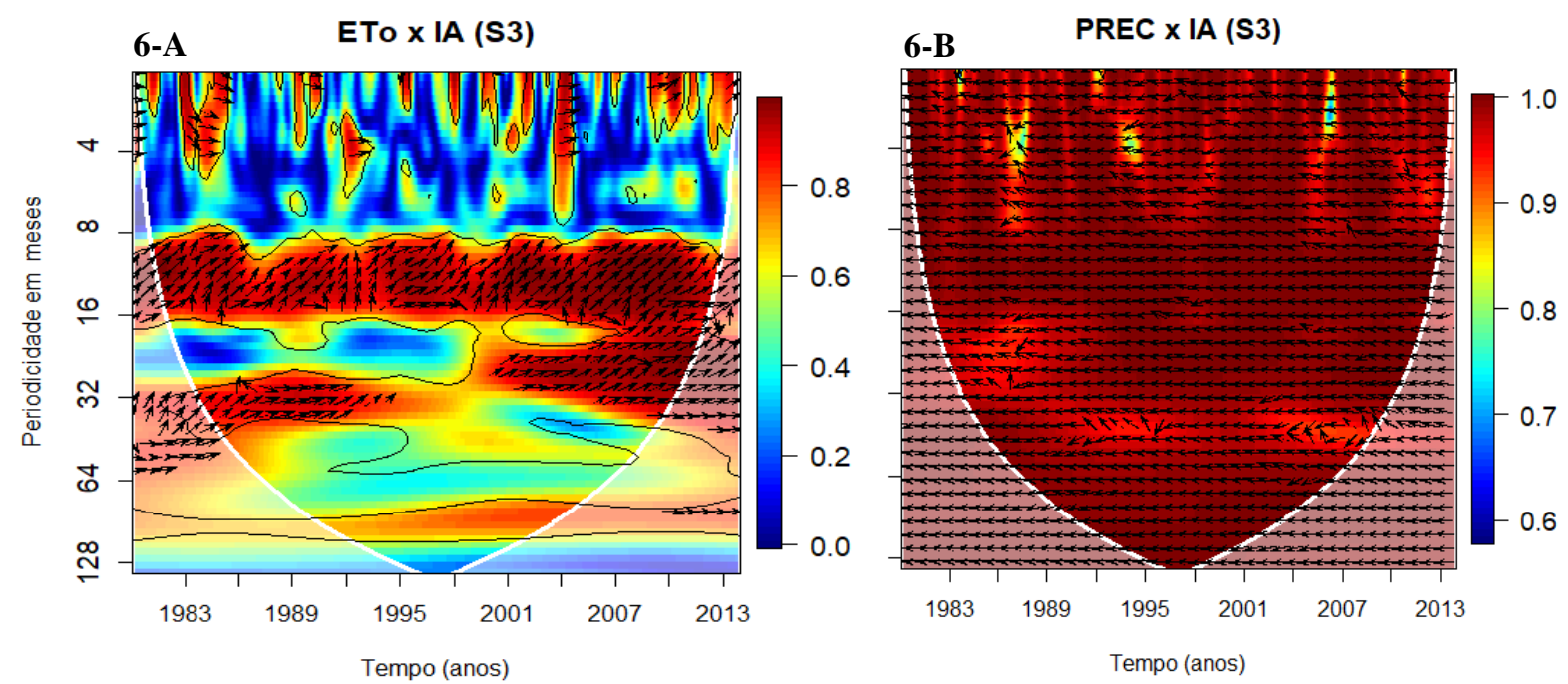

Figura 6. Oscilação da periodicidade (Wavelet Morlet) das correlações existentes entre $\mathrm{ET}_{0} \mathrm{x}$ IA (6A) e Precipitação x IA (6B) para a Sub-região 3 (S3) da ET, no Nordeste do Brasil, entre 1980 e 2013. Fonte: Elaboração dos autores.

Identificou-se ainda que o IA depende predominantemente da precipitação, e que no período das chuvas o IA expressa uma diminuição (Figura 6-B). Entretanto para a relação $\mathrm{ET}_{0}$ e IA (Figura 6-A), não foi observada a mesma predominância em todas as periodicidades, isso reflete na fundamentação da equação preconizada por Thornthwaite (1948), uma vez que a precipitação acumulada mensal for igual a zero o IA será sempre igual a um, independente do aumento ou diminuição da $\mathrm{ET}_{0}$. Portanto, é por isso que na sub-região escolhida (S3), o período praticamente sem chuvas (inverno/primavera) ocorre nas periodicidades entre 4 e 8 meses (Figura 6-A), que culminou numa correlação fraca entre a $\mathrm{ET}_{0}$ e IA.

\section{CONCLUSÕES}

De acordo com os resultados encontrados neste trabalho, conclui-se que:

1. O NEB apresenta 5 sub-regiões de acordo com os valores médios mensais da $\mathrm{ET}_{0}$;

2. A S3 é a sub-região que apresenta os maiores valores médios de $\mathrm{ET}_{0}$ mensais $(\sim 174,8 \mathrm{~mm})$ e anuais $(2098 \mathrm{~mm})$, sendo observados também as maiores variabilidades $(\mathrm{CV}=7,9 \%)$. Por outro lado, a S5 apresentou os menores valores de $\mathrm{ET}_{0}$ (de abril a outubro), média de 99,0 mm, e anualmente (1362,8 mm) apresentando-se a menor dispersão (5\%);

3. Em ordem decrescente os valores médios dos acumulados anuais de $\mathrm{ET}_{0}$, por sub-região no NEB foram para a S3 (2098,0 mm), S2 (1736,9 mm), S4 (1643,5 mm), S1 (1489,3 mm) e S5 (1362,8); 
4. O IA no NEB é predominantemente maior em SON (primavera), quando os valores médios das $E_{0}$ 's superam os das precipitações. Nas demais estações do ano, as precipitações influenciadas pelos respectivos sistemas meteorológicos vigoram para a diminuição do IA;

5. As periodicidades, por meio da análise de Wavelet, permitiram determinar que a variável dominante para o IA é a precipitação, e que no período sem chuvas (periodicidade de 4 a 8 meses), a ET 0 não apresenta correlações altas com o IA, uma vez que a precipitação sendo igual a zero, independente do aumento ou diminuição da ET 0 , o IA será igual a 1.

\section{REFERÊNCIAS}

ALLEN, R. G.; PEREIRA, L. S.; RAES, D.; SMITH, M. Crop evapotranspiration: guidelines for computing crop water requirements. (FAO. Irrigation and Drainage Paper, 56). Rome: FAO, 300 p. 1998.

ALMEIDA, H.A.; CABRAL JÚNIOR, J.B. Variabilidades Sazonais e Interdecadais da Chuva nas Microrregiões Geográficas do Estado da Paraíba. Revista Brasileira de Geografia Física, v.7, n.5, p.846-858, 2014.

AMBRIZZI, T.; ROCHA, R.P.; MARENGO, J.A.; PISNITCHENCO, I.; NUNES, L.A.; FERNANDEZ, J.P.R. Cenários regionalizados de clima no Brasil e América do Sul para o Século XXI: Projeções de clima futuro usando três modelos regionais. Relatório 3. CPTEC/INPE; IAG/ USP; FBDS. Ministério do Meio Ambiente. Secretaria de Biodiversidade e Florestas. Diretoria de Conservação da Biodiversidade. 108 pp, 2007.

AMORIM, A.C.B.; CHAVES, R.R.; SILVA, C.M.S. Influence of the tropical Atlantic Ocean's sea surface temperature in the Eastern Northeast Brazil precipitation. Atmospheric and Climate Sciences, v. 4, n. 05, p. 874-883, 2014.

BRASIL, MI - Ministério da Integração. Resolução n⿳0 115, 23/ 11/ 2017. Aprova a Proposição $n^{\circ} 113 / 2017$, que acrescenta municípios a relação aprovada pela Resolução CONDEL n ${ }^{\circ} 107$, de 27 de julho de 2017. Brasília: Diário Oficial da União, 5 de dezembro de 2017, p. 32. Disponível em: sudene.gov.br/images/arquivos/semiarido/arquivos/resolucao115-23112017delimitacaodosemiarido-DOU.pdf. Acesso em: 01/2018.

BRITO, S. S. B.; CUNHA, A. P. M. A.; CUNNINGHAM, C. C.; ALVALÁ, R.C; MARENGO, J.A.; CARVALHO, M.A Frequency, duration and severity of drought in the Semiarid Northeast Brazil region. International Journal of Climatology, 2017.

CABRAL JÚNIOR, J.B. Estimativas, variabilidade e análise de tendência da evapotranspiração de referência em perímetros irrigados, no semiárido do nordeste brasileiro. 111 p., Dissertação (Mestrado em Ciências Climáticas) - Programa de Pós-Graduação em Ciências Climáticas. Universidade Federal do Rio Grande do Norte, Natal-RN, 2015.

CABRAL JÚNIOR, J.B.; SANTOS, C. M.; ALMEIDA, H. A. Comparação mensal, sazonal e anual de métodos de estimativas da evapotranspiração de referência para Juazeiro-BA e Petrolina-PE. Revista de Geociências do Nordeste, v. 3, n. 2, p. 23-42, 2017. 
CHEN, H.; GUO, S.L.; XU, C.Y.; SINGH, V.P. Historical temporal trends of hydro-climatic variables and runoff response to climate variability and their relevancein water resource management in the Hanjiang basin. Journal of Hydrology, v.344, n.3-4, p.171-184, 2007.

CEPED/UFSC. Atlas brasileiro de desastres naturais: 1991 a 2012 / Centro Universitário de Estudos e Pesquisas sobre Desastres. 2. ed. rev. ampl. - Florianópolis: CEPED UFSC, 2013. Volume Rio Grande do Norte. 126 p.

CUNHA, A. P. M.; AlvalÁ, R. C.; NOBRE, C. A.; CARVAlhO, M. A. Monitoring vegetative drought dynamics in the Brazilian semiarid region. Agricultural and Forest Meteorology, v. 214-215, p. 494-505, 2015.

DROGUE, G.; PFISTER, L.; LEVIANDIER, T.; IDRISSI, A.E.; IFFLY, J.F.; MATGEN, P.; HUMBERT, J.; HOFFMANN, L. Simulating the spatio-temporal variability of streamflowresponse to climate change scenarios in a mesoscale basin. Journal of Hydrology, v.293, n.1-4, p.255-269, 2004.

FENG, Y.; CUI, N.; ZHAO, L.; GONG, D.; ZHANG, K. Spatiotemporal variation of reference evapotranspiration during 1954-2013 in Southwest China. Quaternary International, v. 441, p. 129-139, 2017.

FERREIRA, A.G.; MELLO, N.G.S. Principais sistemas atmosféricos atuantes sobre a região Nordeste do Brasil e a influência dos oceanos Pacífico e Atlântico no clima da região. Revista Brasileira de Climatologia, v. 1, n. 1, 2005.

HAN, S.J.; TANG, Q.H.; XU, D.; WANG, S.L. Irrigation-induced changes in potential evaporation: more attention is needed. Hydrological Processes, v.28, n.4, p.2717-2720, 2014.

HAN, S.J.; HU, H.P. Spatial variations and temporal changes in potential evaporation in the Tarim Basin, northwest China (1960-2006): influenced by irrigation? Hydrological Processes, v.26, n.20, p.3041-3051, 2012.

HASTENRATH, S. Exploring the climate problems of Brazil's Nordeste: A review. Climatic Change, v. 112, n. 2, p. 243-251, 2012.

HASTENRATH, S. Prediction of Northeast Brazil rainfall anomalies. Journal of Climate, v. 3, n. 8, p. 893-904, 1990.

IBGE - Instituto Brasileiro de Geografia e Estatística. Mapa de biomas do Brasil. Rio de Janeiro, 2004.

IBGE - Instituto Brasileiro de Geografia e Estatística. BRASIL. Disponível em: <http://www.ibge.gov.br/home/geociencias/areaterritorial/principal.shtm>. Acesso em: 14 set 2017.

IPCC, 2007. Climate Change 2007: the Physical Science Basis, Summary for Policymakers.Cambridge University Press, Cambridge.

JESUS, E.S.; MATTOS, A. Análise espaço temporal da evapotranspiração sobre a microrregião do Seridó no estado do Rio Grande do Norte. Holos, v. 6, p. 22-32, 2013. 
KELLER FILHO, T.K.; ASSAD, E.D.; LIMA, R.S.R. Regiões pluviometricamente homogêneas no Brasil. Pesquisa Agropecuária Brasileira, vcl.40, n.4, p.311-322, 2005.

KEYANTASH, J.; DRACUP, J.A. The quantification of drought: an evaluation ofdrought indices. Bulletin of the American Meteorological Society, v. 83, n. 8, p. 1167-1180, 2002.

LIU, X.; ZHANG, D.; LUO, Y.; LIU, C. Spatial and temporal changes in aridity index in northwest China: 1960 to 2010. Theoretical and applied climatology, v.112, n.1-2, p.307316, 2013.

MARENGO, J. A.; BERNASCONI, M. Regional differences in aridity/drought conditions over Northeast Brazil: present state and future projections. Climatic Change, v. 129, n. 1-2, p. 103$115,2015$.

MARENGO, J. A.; TORRES, R. R.; ALVES, L. M. Drought in Northeast Brazil—past, present, and future. Theoretical and Applied Climatology, v. 129, n. 3-4, p. 1189-1200, 2017.

MARENGO, J. A.; ALVES, L. M.; BESERRA, E. A.; LACERDA, F. F. Variabilidade e mudanças climáticas no semiárido brasileiro. Campina Grande-PB, 2011.

MARES, M.A.; WILLIG, M.R.; LACHER, J.R.; T.E. The Brazilian Caatinga in South American zoogeography: tropical mammals in a dry region. Journal of Biogeography, v.12, n.1, p. 57-69, 1985.

MCKEE, T.B.; DOESKEN, N.J.; KLEIST J. The relationship of drought frequency and duration to time scales. In: Proceedings of the 8th Conference on Applied Climatology. Boston, MA: American Meteorological Society, 1993. p. 179-183.

MINGOTI, S. A. Análise de Dados Através de Métodos de Estatística - Multivariada - Uma Abordagem Aplicada. UFMG, 2005.

MIMMACK, G. M.; MASON, S. J.; GALPIN, J. S. Choice of distance matrices in cluster analysis: defining regions. Journal of Climate, v. 14, p. 2790-2797, 2001.

Ministério da Integração Nacional (MI) - Brasil. Programa Água para todos. Disponível em: http://www.mi.gov.br/. Acessado em 15 de dez de 2017.

MOLION, L.C.B.; BERNARDO, S.O. Uma revisão da dinâmica das chuvas no nordeste brasileiro. Revista Brasileira de Meteorologia, v. 17, n. 1, p. 1-10, 2002.

NIMER, E. Climatologia do Brasil, Fundação Instituto Brasileiro de Geografia e Estatística: Rio de Janeiro, Brasil, 1989.

NOBRE, P.; MARENGO, J.A.; CAVALCANTI, I. F. A.; OBREGON, G. Seasonal-todecadal predictability and prediction of South American climate. Journal of climate, v. 19, n. 23, p. 5988-6004, 2006.

OLIVEIRA, P.T.; SILVA, C.M.S.; LIMA, K.C. Linear trend of occurrence and intensity of heavy rainfall events on Northeast Brazil. Atmospheric Science Letters, v. 15, n. 3, p. 172177, 2014a. 
OLIVEIRA, P.T.; LIMA, K.C.; SILVA, C.M. S. Synoptic environment associated with heavy rainfall events on the coastland of Northeast Brazil. Advances in Geosciences, v. 35, p. 73-78, $2013 b$.

OLIVEIRA, N.F.; SILVA, M.T.; GONÇALVES, A.A.S.; ARAÚJO, R.M.S. Variabilidade espaço temporal da Evaporação Potencial no submédio do rio São Francisco utilizando a análise fatorial em componentes principais. Ciência e Natura, v. 36, n. II, 2014.

PAREDES, F. J.; BARBOSA, H. A.; GUEVARA, E. Spatial and temporal analysis of droughts in northeastern Brazil. Agriscientia, v. 32, p. 1-14, 2015.

REBOITA, M.S.; RODRIGUES, M.; ARMANDO, R.P.; FREITAS, C.; MARTINS, D.; MILlER, G. Causas da Semi-Aridez do Sertão Nordestino. Revista Brasileira de Climatologia, v.19, 2016.

REBOITA, M.S.; GAN, M.A.; ROCHA, R.P.; AMBRIZZI, T. Regimes de precipitação na América do Sul: uma revisão bibliográfica. Revista Brasileira de Meteorologia, v. 25, n. 2, 2010.

SANTOS, W.O.; BATISTA, B.D.O.; ASSIS, J.P.; RODRIGUES, W. M.; SOBRINHO, J. E. Métodos de estimativa da evapotranspiração de Referência $\left(\mathrm{ET}_{0}\right)$ para a região de MossoróRN. Revista Verde de Agroecologia e Desenvolvimento Sustentável, v. 5, n. 5, p. 210-221, 2010.

SAPIR, D.G., BELOW, R., EM-DAT: International Disaster Database. Université Catholique de Louvain, Brussels, Belgium www.emdat.be, 2014.

SHAN, N.; SHI, Z.; YANG, X.; GAO, J.; CAI, D. Spatiotemporal trends of reference evapotranspiration and its driving factors in the Beijing-Tianjin Sand Source Control Project Region, China. Agricultural and Forest Meteorology, v. 200, 2015.

SILVA, V. D. P. R. On climate variability in Northeast of Brazil. Journal of Arid Environments, v. 58, n. 4, p. 575-596, 2004.

SOUZA, P.; CAVALCANTI, I.F. Albuquerque. Atmospheric centres of action associated with the Atlantic ITCZ position. International Journal of Climatology, v. 29, n. 14, p. 2091-2105, 2009.

THORNTHWAITE, C.W. An approach toward a rational classification of climate. Geographical Review, v.38, n.1, p.55-94, 1948.

TIMM, N. H. Applied Multivariate Analysis. New York: Springer Verlag, 2002. TRMM (Tropical Rainfall Mission Mission). Disponível em: <http://trmm.gsfc.nasa.gov>. Acesso em: 22/08/2017.

TORRENCE, C.; WEBSTER, P.J. Interdecadal changes in the ENSO-monsoon system. Journal of Climate, v. 12, n. 8, p. 2679-2690, 1999.

TRENBERTH, K. E.; DAI, A.; VAN DER SCHRIER, G.; JONES, P. D.; BARICHIVICH, J.; BRIFFA, K. R.; SHEFFIELD, J. Global warming and changes in drought. Nature Climate 
Change, v. 4, n. 1, p. 17-22, 2014.

UVO, C.B. A Zona de Convergência Intertropical (ZCIT) e sua relação com a precipitação da Região Norte do Nordeste Brasileiro. 1989. 99f. Dissertação (Mestrado em meteorologia) Instituto Nacional de Pesquisas Espaciais, São José dos Campos, 1989.

WANG, L.; CAO, L.; DENG, X.; JIA, P.; ZHANG, W.; XU, X.; ... \& CHEN, Y. Changes in aridity index and reference evapotranspiration over the central and eastern Tibetan Plateau in China during 1960-2012. Quaternary International, v. 349, p. 280-286, 2014.

WANG, W.; ERTSEN, M. W.; SVOBODA, M. D.; HAFEEZ, M. Propagation of drought: From meteorological drought to agricultural and hydrological drought. Advances in Meteorology, v. 2016, n. 4, 2016.

WANG, Z.; XIE, P.; LAI, C.; CHEN, X.; WU, X.; ZENG, Z.; LI, J. Spatiotemporal variability of reference evapotranspiration and contributing climatic factors in China during 1961-2013. Journal of Hydrology, v. 544, p. 97-108, 2017.

WARD, J. Hierarchical Grouping to optimize an objective function. Journal of the American Statistical Association, v. 58, p. 236-244, 1963.

XAVIER, A. C.; KING, C. W.; SCANLON, B. R. Daily gridded meteorological variables in Brazil (1980-2013). International Journal of Climatology, v. 36, n. 6, p. 2644-2659, 2015.

XAVIER, T.M.B.S. Tempo de Chuva - In Estudos Climáticos e de Previsão para o Ceará e Nordeste Setentrional, cap. 36, 6, 11. ABC Editora, Fortaleza-Ceará, 2001, 478 p., 2001.

XU, Y.; XU, Y.; WANG, Y.; WU, L.; LI, G.; SONG, S. Spatial and temporal trends of reference crop evapotranspiration and its influential variables in Yangtze River Delta, eastern China. Theoretical and Applied Climatology, v. 130, n. 3-4, p. 945-958, 2017.

ZHANG, K. X.; PAN, S. M.; ZHANG, W.; XU, Y. H.; CAO, L. G.; HAO, Y. P.; WANG, Y. Influence of climate change on reference evapotranspiration and aridity index and their temporal-spatial variations in the Yellow River Basin, China, from 1961 to 2012. Quaternary International, v. 380-381, p. 75-82, 2015.

\section{AGRADECIMENTOS}

O primeiro autor agradece à Coordenação de Aperfeiçoamento de Pessoal de Nível Superior (CAPES) pela bolsa de Doutorado durante 2015 a 2018.

Recebido em: 23/06/2018

Aceito para publicação em: 29/06/2018 